\title{
A Case Report of Splenic Tuberculous Abscess
}

\author{
Manoj Kumar Sah*, Bhawesh Thapa, Samrat Shrestha
}

Department of Medicine, NAMS, Bir Hospital, Kathmandu, Nepal

\section{DOI Name}

http://dx.doi.org/10.3126/jaim.v7i1.19579

Keywords

Splenic tuberculosis, abscess

\section{Citation}

Manoj Kumar Sah, Bhawesh Thapa, Samrat Shrestha. A Case Report of Splenic Tuberculous Abscess. Journal of Advances in Internal Medicine 2017;06(01):23-25

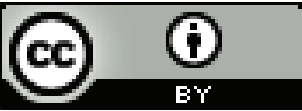

This work is licensed under a Creative Commons Attribution 3.0 Unported License.

\begin{abstract}
Tuberculosis is a common infectious disease in South-Asian region which can involve different organs. Splenic tuberculosis is a rare clinical entity. Here we report a case of tuberculous abscess of spleen in a 72 year old diabetic male. It was disseminated type, it was confirmed by ultrasonography guided aspiration of abscsess for gene $x$-pert test, which was positive. Patient was given antitubercular treatment after the confirmation of the diagnosis and is improving with given treatment.
\end{abstract}

\section{INTRODUCTION}

\section{Introduction}

Tuberculosis is oldest diseases known to affect humans and major cause of death worldwide. ${ }^{1}$ In 2015, there were an estimated 10.4 million new TB cases worldwide and people living with HIV accounted for 1.2 million (11\%) of all new TB cases. WHO estimates incidence of 156 cases per 1,00,000 cases of tuberculosis in Nepal for 2015. ${ }^{2}$ Extrarapulmonary TB constit utes $15-20 \%$ of all cases; abdominal TB accounts for $3 \%$ involving particularly ileocaecal region, mesenteric lymph nodes and peritoneum. ${ }^{3}$ Splenic tuberculosis occurs mostly as part of miliary tuberculosis in immunocompromised patients. Patients susceptible to acquiring splenic tuberculosis usually have one of the following risk factors: immunosuppression, preceding pyogenic infections, splenic abnormalities, prior trauma to the spleen, sickle cell disease and in the immunocompetent patient another body site infected by tuberculosis. ${ }^{4}$

Tuberculosis can be diagnosed by different methods. GeneXpert is one of the modalities of diagnosing TB. GeneXpert assay is an automated molecular test for Mycobacterium tuberculosis (MTB) and resistance to rifampin (RIF). It uses heminested real-time polymerase chain-reaction (PCR) assay to amplify an MTB-specific sequence (192 bp segment) of the rpoB gene, which is probed with molecular beacons for mutations within the rifampin-resistance determining region. In various published studies, GeneXpert sensitivity have ranged from $25.0 \%$ to $95.1 \%$ for EPTB specimens ${ }^{5}$.

\section{Case Report}

A 72 year old male from Terai Region of Nepal is a known case of Hypertension and Diabetes Mellitus type 2, under regular medication for last 10 years. He is a non alcoholic and an exsmoker who left smoking 16 years back, amounting around 25 pack years. He presented with chief complaints of loss of appetite with loss of weight around $20 \%$ of his body weight in last 5 months. It was associated with moderate to high grade, intermittent fever for last 3 months. It was associated with chills and rigors which resolved only transiently after treatment received at many health care facilities. Patient had undergone various blood and radiological investigations at other centres

\footnotetext{
* Corresponding author Dr Manoj kumar shah.

Associate professor of Internal medicine. Consultant Physician, NAMS. Bir Hospital. Email address-najmanas@gmail.com
} 
and provisional diagnosis of Splenic Abscess with Pancytopenia with type II Diabetes Mellitus with Hypertension was made. He was managed accordingly but he did not improve despite medications and was referred to our center.

On presentation at our center, physical examination revealed ill looking patient with vitals within normal limits, pallor, tenderness over the left hypochondriac region with no palpable organomegaly clinically. Hematological investigations showed picture of pancytopenia with erythroid sedimentation of $57 \mathrm{~mm}$ in $1^{\text {st }}$ hour. Chest X-ray showed blunting of right costophrenic angle with homogenous opacity over the right lower zone. Ultrasonography showed enlarged spleen of $13.5 \mathrm{~cm}$ with a complex cystic space occupying lesion of around $42 \times 37$ $\mathrm{mm}^{2}$ with internal echoes (abscess). Sputum examination for AFB and GeneXpert did not detect tuberculosis. Computed tomography showed hepatosplenomegaly of $17 \mathrm{~cm}$ and $13.9 \mathrm{~cm}$ respectively with multiple non enhancing hypodense areas in spleen measuring $36 \times 32 \times 32 \mathrm{~mm}^{3}$ likely splenic abscess, dilated splenic vein with tortuous few collaterals and ascites.
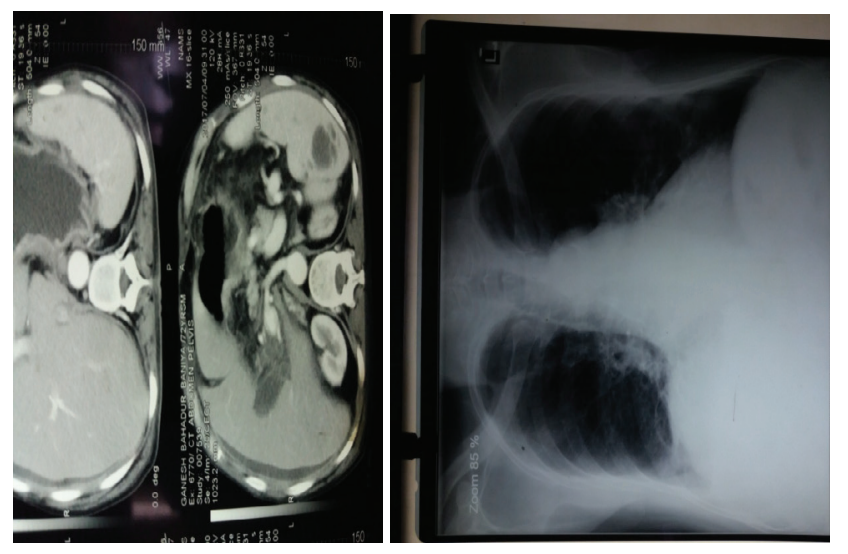

A clinical diagnosis of pancytopenia under evaluation with splenic abscess with right sided pleural effusion with type II diabetes mellitus with hypertension was made and managed accordingly. Investigations for infectious disease revealed no positive results. Echocardiography revealed Mild MR, AR, TR, Mild Pulmonary Arterial hypertension with PASP $38 \mathrm{~mm}$ of $\mathrm{Hg}$, Diastolic LV dysfunction grade-I and LVEF 65\%. Bone marrow examination showed hypocellular marrow with mild erythroid hyperplasia. Ascitic fluid analysis revealed Low SAAG (0.8) and high protein $(4 \mathrm{gm} / \mathrm{dl})$ and ADA of $92.2 \mathrm{U} / \mathrm{L}$. Aspiration of the pus under ultrasonographic guidance was done. Pus on Gene Xpert revealed Rifampicin sensitive Tuberculosis however AFB stain showed no AFB. Currently, patient is kept on antitubercular treatment (ATT) Category I as per WHO Regimen and is improving with treatment.

\section{Discussion}

Tuberculosis of the spleen was first described in literature in 1846 by Coley referring to enlarged spleen secondary to tuberculosis with absent or limited involvement of other organs. ${ }^{2}$ The common forms of extra pulmonary tuberculosis are lymphadenopathy, pleural effusion, bone and joint disease, intestinal disease, meningitis and miliary disease. ${ }^{7}$ Majority of cases of Splenic tuberculosis are described in immunocompromised patients. Only sporadic cases are reported in immunocompetent patients. ${ }^{2}$ The incidence of splenic abscess is very low $(0.14$ to $0.7 \%$ ) in various autopsy studies, and is usually associated with septicaemic conditions. Splenic tuberculosis usually occurs following the haematogenous spread of infection, occasionally due to contiguous spread of infection, or, as a part of disseminated disease. ${ }^{6}$

Splenic tuberculosis patients can also present with hematologic abnormalities. Usually they are cytopenic, but cases of polycythemia have also been reported ${ }^{1}$. Antituberculous medications are the first line of treatment. Splenectomy is indicated as a mode of treatment under particular conditions like failure of medical treatment, cytopenia or polycythemia, tuberculous splenomegaly with gastrointestinal bleeding secondary to portal hypertension, failure of percutaneous abscess drainage, and multiple splenic abscesses. ${ }^{7}$ No reduction in size of the abscess and persistent symptoms at approximately 3months follow-up is also considered as a therapeutic failure that needs surgical intervention. ${ }^{7}$ In a study from India, the positivity rate with GeneXpert assay was $24.6 \%$ for CSF, 32\% for pleural fluid and $27.8 \%$ for ascitic fluid. Another study from Pakistan have reported $15.8 \%, 40.0 \%$ and $6.3 \%$ respectively of pleural fluid, CSF and ascitic fluids samples to be positive ${ }^{5}$.

Zulkarnaen AN. Splenic tuberculosis presenting as pyrexia of unknown origin. Med J Malaysia 2007; 62 : 70-1.

4. A case of isolated splenic tuberculosis Johanna V. Basa International Journal of Surgery Case Reports 8 (2015) 117-119

5. Use of GeneXpert Assay for Diagnosis of Tuberculosis 
From Body Fluid Specimens, a 2 Years Study, Journal of Microbiology \& Biotechnology ,Volume 1 Issue 1, 2016

6. Rajesh S. Shinde Tuberculous Splenic Abscess Bombay Hospital Journal, Vol. 54, No. 1, 2012

7. Sudarshan P B A rare case of splenic tuberculosis,
MedPulse - Internation Medical Journal, ISSN: 23482516, EISSN: 2348-1897, Volume 2, Issue 5, May 2015

8. Tubercular Abscess of the spleen in a child: Conservative Management and Long-Term Follow-up Devi Dayal et al Journal of pediatric Infectious Diseases July 2017 Researchgate. 